\title{
CLINICOETIOLOGICAL PROFILE OF RESPIRATORY DISTRESS IN NEONATES
}

\author{
Reshmi1 ${ }^{1}$ Manjunathaㄹ, Sagar Bharamakkanavar ${ }^{3}$
}

${ }_{1}^{1}$ Senior Resident, Department of Paediatrics, Christian Medical College, Vellore, Tamilnadu, India.

${ }^{2}$ Associate Professor, Department of Paediatrics, Cheluvamba Hospital, MMC\&RI, Mysore, Karnataka, India.

${ }^{3}$ Resident, Department of Paediatrics, Cheluvamba Hospital, MMC\&RI, Mysore, Karnataka, India.

\begin{tabular}{l}
\hline ABSTRACT \\
BACKGROUND \\
Respiratory distress is the most common cause of morbidity and mortality in newborn. In the last 3 decades, several improvements \\
have been introduced to reduce the incidence, severity, and mortality of neonatal respiratory distress. The objectives of the study \\
were- 1. to estimate the proportion of various aetiology of respiratory distress among neonates born in Cheluvamba hospital. 2 . to \\
investigate the maternal and perinatal risk factors for the development of respiratory distress. 3. to determine the immediate \\
outcome of neonates with respiratory distress admitted in the NICU.
\end{tabular}

\section{MATERIALS AND METHODS}

This was a cross sectional descriptive study conducted among neonates admitted with respiratory distress to the NICU of Cheluvamba hospital attached to Mysore Medical College from January 2015 to June 2016. A total of 250 neonates were included. Maternal details were collected from the mothers' case records. Intrapartum details were documented. Baby's birth weight, sex and gestational age were noted. Respiratory distress was defined as presence of tachypnoea, chest retraction and grunting, any two of the three.

\section{RESULTS}

Among the various aetiologies, majority were meconium aspiration syndrome 78 cases $(31.2 \%)$, followed by hyaline membrane disease 65 cases (26\%). There was significant male predilection (M: $\mathrm{F}=1.3: 1$; $\mathrm{p}<0.023$ ). Majority were between 2.5-3.5 Kgs. 46.8\% (117 cases). Majority were term 57.6\% (144 cases). There was no significant association of severity of respiratory distress and maternal age. Majority were primi mothers, $60.8 \%(\mathrm{p}<0.005)$. Maternal fever or urinary tract infection were in 17 cases, of which $58.82 \%$ had positive sepsis screen. There were 11 diabetic mothers who predisposed to either TTNB or HMD (p<0.006). Among 47 mothers with pre-eclampsia, HMD was 31.91\% followed by MAS 25.53\% ( $<<0.001$ ). Among twins, majority had HMD $82.35 \%$ (p < 0.004). Of 101 cases with meconium stained liquor, 78 cases (77.23\%) developed meconium aspiration syndrome. 18 cases had prolonged rupture of membranes of which 11 cases had culture positive sepsis. Majority were born of normal vaginal delivery 147 cases (58.8\%). The neonates with respiratory distress had a survival rate of $75.2 \%$ in our NICU. Majority who had expired were due to hyaline membrane disease $53.3 \%$ (33 out of 62 cases). Surgical causes of respiratory distress had highest case fatality (100\%) followed by hyaline membrane disease (50.8\%).

\section{CONCLUSION}

Meconium aspiration syndrome is the most common cause of respiratory distress among neonates born in Cheluvamba hospital followed by hyaline membrane disease. Neonatal respiratory distress had a male predilection. Maternal risk factors which were significant were primi parity, gestational diabetes, pre-eclampsia, multiple gestation, not receiving antenatal steroids in preterm labour. Intrapartum risk factors like prolonged labour, meconium stained amniotic fluid mode of delivery and low Apgar scores at birth also affects the aetiology of respiratory distress in neonates. Mortality among the neonates with respiratory distress was $24.8 \%$. The highest case fatality rate is for hyaline membrane disease $50.8 \%$.

\section{KEY WORDS}

Respiratory Distress Syndrome, Meconium Aspiration Syndrome, Pneumonia

HOW TO CITE THIS ARTICLE: Reshmi, Manjunatha, Bharamakkanavar S. Clinicoetiological profile of respiratory distress in neonates. J. Evolution Med. Dent. Sci. 2019;8(12):889-892, DOI: 10.14260/jemds/2019/198

\section{BACKGROUND}

Respiratory distress is one of the most common disorders encountered within the first 48-72 hours of life and is a major cause for the leading morbidity in newborn, especially in preterm neonates. Respiratory distress occurs approximately 'Financial or Other Competing Interest': None.

Submission 23-01-2019, Peer Review 07-03-2019,

Acceptance 13-03-2019, Published 25-03-2019.

Corresponding Author:

Dr. Manjunatha,

Associate Professor,

Department of Paediatrics, Cheluvambha Hospital,

MMC\&RI, Mysore-570001, Karnataka, India.

E-mail:manjunatha0505@gmail.com

DOI: $10.14260 /$ jemds $/ 2019 / 198$

\section{(c) (1) $(9)$}

in 7 percent of neonates and preparation is crucial for physicians providing neonatal care. ${ }^{1}$

In the last 3 decades several improvements have been introduced to reduce the incidence, severity and mortality of neonatal respiratory distress. Among these milestones are the induction of lung maturation with antenatal steroids, centralization of high-risk pregnancies to tertiary perinatal centres, usage of surfactant to treat immature lungs and introduction of new methods of mechanical ventilation and the use of nasal CPAP.2

\section{MATERIALS AND METHODS}

\section{Source of Data}

This study was a cross sectional descriptive study conducted by observation of neonates admitted with respiratory 
distress to the NICU of Cheluvamba hospital attached to Mysore Medical College from January 2015 to June 2016.

\section{Sampling Technique}

Convenient sampling.

\section{Study Design}

Cross sectional descriptive study.

\section{Inclusion Criteria}

Neonates born in Cheluvamba hospital with presence of at least 2 of the following criteria-

- Tachypnoea (RR> 60 per min)

- Subcostal or intercostals recessions

- $\quad$ Expiratory grunting/groaning ${ }^{3}$

\section{Exclusion Criteria}

- Neonates with respiratory distress who were born outside our hospital and was referred to us for management of respiratory distress. ${ }^{4}$

- Babies born in our hospital but developing respiratory distress after 28 days of Life. ${ }^{5}$

\begin{tabular}{|c|c|c|c|c|c|c|c|c|c|}
\hline \multirow{2}{*}{ Diagnosis } & \multicolumn{3}{|c|}{ Preterm } & \multicolumn{3}{|c|}{ Term } & \multicolumn{3}{|c|}{ Post Term } \\
\hline & SGA & AGA & LGA & SGA & AGA & LGA & SGA & AGA & LGA \\
\hline Meconium Aspiration Syndrome & $20.8 \%$ & $52 \%$ & $10.4 \%$ & $104 \%$ & $5722.8 \%$ & 0 & $20.8 \%$ & $20.8 \%$ & 0 \\
\hline Hyaline Membrane Disease & $83.2 \%$ & $5722.8 \%$ & 0 & $93.6 \%$ & 0 & 0 & 0 & 0 & 0 \\
\hline Transient Tachypnoea & $10.4 \%$ & $135.2 \%$ & $10.4 \%$ & 0 & $249.6 \%$ & $20.8 \%$ & 0 & 0 & 0 \\
\hline Pneumonia & 0 & $41.6 \%$ & $20.8 \%$ & $20.8 \%$ & $176.8 \%$ & 0 & 0 & 0 & 0 \\
\hline Birth Asphyxia & 0 & $62.4 \%$ & 0 & 0 & $176.8 \%$ & 0 & 0 & $10.4 \%$ & 0 \\
\hline Right Pulmonary Aplasia & 0 & 0 & 0 & 0 & $10.4 \%$ & 0 & 0 & 0 & 0 \\
\hline Congenital Diaphragmatic Hernia & $10.4 \%$ & 0 & 0 & $10.4 \%$ & $10.4 \%$ & 0 & 0 & 0 & 0 \\
\hline Tracheo Oesophageal Fistula & 0 & 0 & 0 & $10.4 \%$ & $10.4 \%$ & 0 & 0 & 0 & 0 \\
\hline Congenital Heart Disease & 0 & 0 & 0 & 0 & $10.4 \%$ & 0 & 0 & 0 & 0 \\
\hline Pierre Robin Sequence & 0 & 0 & 0 & 0 & $10.4 \%$ & 0 & 0 & 0 & 0 \\
\hline
\end{tabular}

\section{Table 3. Gestational Age Wise Distribution of Aetiology of Respiratory Distress}

Majority of mothers whose newborns developed respiratory distress were primi mothers $60.8 \%$ (152 cases). Majority of cases of MAS, birth asphyxia and pneumonia were seen in primi mothers.

\begin{tabular}{|c|c|c|}
\hline Diagnosis & Primipara & Multipara \\
\hline MAS & 51 & 27 \\
\hline HMD & 32 & 33 \\
\hline TTNB & 30 & 20 \\
\hline Pneumonia & 17 & 8 \\
\hline Birth Asphyxia & 21 & 3 \\
\hline CDH & 0 & 3 \\
\hline TEF & 0 & 2 \\
\hline Congenital Heart Disease & 0 & 1 \\
\hline Right Pulmonary Agenesis & 1 & 0 \\
\hline Pierre Robin Sequence & 0 & 1 \\
\hline Table 4. Maternal Parity Versus Aetiology of Respiratory \\
\multicolumn{2}{|c}{ Distress (p=0.005) } \\
\hline
\end{tabular}

There were total 11 cases whose mothers had history of gestational diabetes out of which 7 were preterms and 4 were term babies. 6 out of 7 preterms developed HMD ( 6 cases). All term babies and 1 late preterm developed TTNB ( 5 cases).

\section{RESULTS}

Among the various aetiologies majority of cases were meconium aspiration syndrome 78 cases $(31.2 \%)$ followed by hyaline membrane disease 65 cases (26\%).

\begin{tabular}{|c|c|c|}
\hline Diagnosis & No. of Cases & Percentage \\
\hline Meconium Aspiration Syndrome & 78 & $31.2 \%$ \\
\hline Hyaline Membrane Disease & 65 & $26 \%$ \\
\hline Transient Tachypnoea & 50 & $20 \%$ \\
\hline Pneumonia & 25 & $10 \%$ \\
\hline Right Pulmonary Aplasia & 1 & $0.4 \%$ \\
\hline \multicolumn{2}{|c|}{ Table 1. Respiratory Causes } \\
\hline
\end{tabular}

\begin{tabular}{|c|c|c|}
\hline Diagnosis & Cases & Percentage \\
\hline Birth Asphyxia & 26 & $10.4 \%$ \\
\hline Congenital Diaphragmatic Hernia & 3 & $1.2 \%$ \\
\hline Tracheo-Oesophageal Fistula & 2 & $0.8 \%$ \\
\hline Congenital Heart Disease & 1 & $0.4 \%$ \\
\hline Pierre Robin Sequence & 1 & $0.4 \%$ \\
\hline
\end{tabular}

Table 2. Non-Respiratory Causes

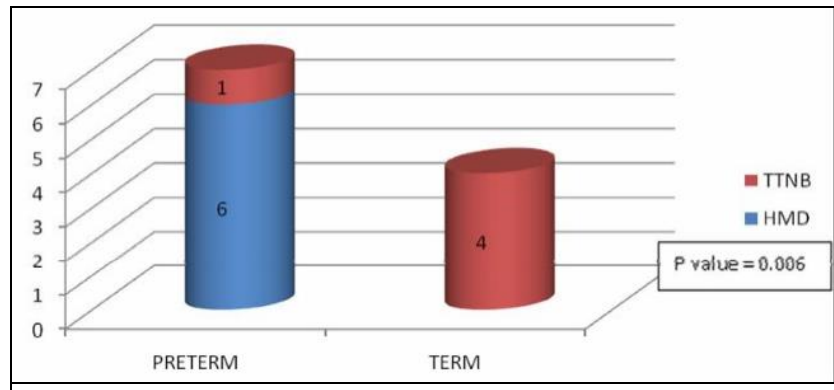

Figure 1. Aetiology of Respiratory Distress in Neonates of Diabetic Mothers 5

Among the non-respiratory causes majority aetiology were birth asphyxia 26 cases (10.4\%).

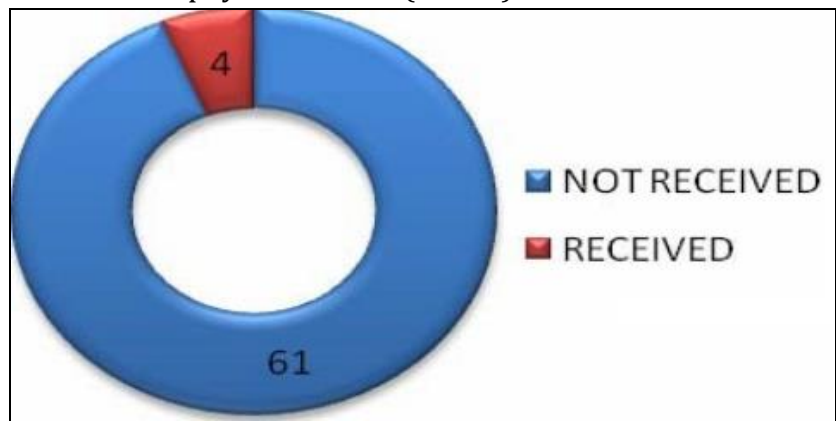

Figure 2. Hyaline Membrane Disease Cases Not Received Antenatal Steroids 
Out of 65 cases of HMD majority 93.85\% (61 out of 65) were cases who have not received antenatal steroids.

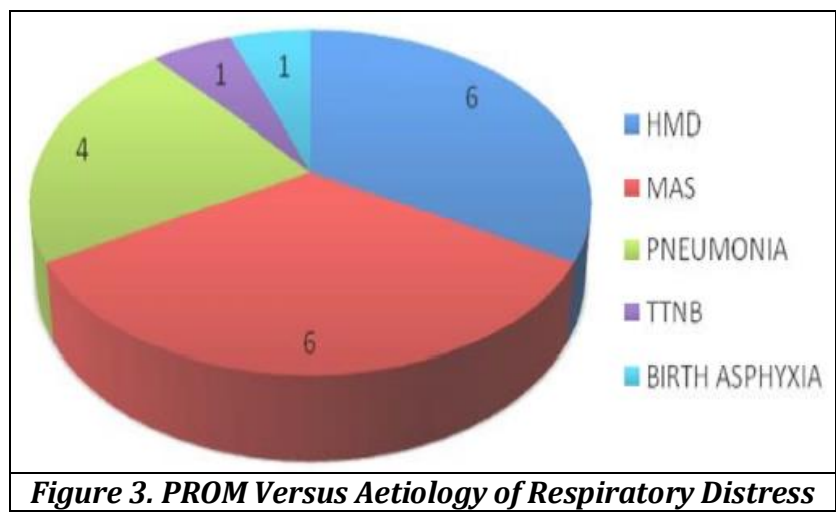

Only 18 cases (7.2\%) had history of prolonged rupture of membranes of which 11 cases $(61.11 \%)$ had culture positive sepsis. 6 cases of HMD, 6 cases of MAS, 4 cases of pneumonia, 1 TTNB, 1 Birth asphyxia.

\section{Immediate Outcome of Respiratory Distress}

The neonates admitted with respiratory distress in our NICU had a survival rate of $75.2 \%$ (188 out of 250). Majority of neonates who expired were due to hyaline membrane disease. $53.3 \%$ ( 33 out of 62 cases).

\section{DISCUSSION}

In the present study, majority of cases of respiratory distress were due to meconium aspiration syndrome followed by hyaline membrane disease. In the study by Clark et $\mathrm{al}^{6}{ }^{6}$ the majority of cases were hyaline membrane disease followed by meconium aspiration syndrome. Dutta et $\mathrm{al}^{7}$ and Kumar et $\mathrm{al}^{8}$ in their study had a highest incidence of transient tachypnoea of newborn. Mathur et al $^{9}$ had pneumonia as the majority of cases. The highest incidence of meconium aspiration syndrome in the present study is probably due to majority of cases of high risk pregnancies being referred to our hospital from the periphery for management and lack of adequate infrastructure to manage the large number of deliveries in our hospital which results in delay in early identification of fetal compromise and timely interventions like elective caesarean section in the presence of risk factors.

In all studies there has been a male predilection for development of respiratory distress the cause of which is unknown. In our study the Male: Female ratio of 1.3:1 which was statistically significant $(p=0.023)$, was similar to that observed by Rubatelli et al. ${ }^{10}$

In the present study, majority of the study population were of birth weight above $2.5 \mathrm{~kg}$, followed by low birth weight neonates. Similar observations were made by Li-ling et al ${ }^{11}$ in 23 NICUs in tertiary maternity and children's hospitals from major cities in China.

In the present study majority of neonates admitted with respiratory distress were term neonates. Similar observations were also made by Dutta et al $^{7}$ in their study among Italian infants born in 65 hospitals in Italy in 1996 and Abdelrahman et al in the NICU of Omdurman Maternity Hospital, Sudan in 2013.

In the present study all cases of respiratory distress among neonates of diabetic mothers were either hyaline membrane disease or transient tachypnoea of newborn. Similar observations were also made by Rubetelli et al and by Li-ling et al.

In the present study the majority of cases of respiratory distress among neonates whose mothers had pre-eclampsia were hyaline membrane disease followed by meconium aspiration syndrome. In the study by Li-ling et al. Majority of cases were hyaline membrane disease followed by pneumonia. The higher incidence of hyaline membrane disease is probably due to the increased chance of preterm births in case of maternal severe pre-eclampsia for which termination of pregnancy is indicated in view of maternal benefits.

In the present study, $93.85 \%$ cases of hyaline membrane disease have not received antenatal steroids whereas in the study by Dani et al ${ }^{12}$ only $25.07 \%$ cases of hyaline membrane disease have not received antenatal steroids. In the present study, we had 101 cases with history of meconium stained amniotic fluid out of which $77.2 \%$ cases developed meconium aspiration syndrome. This was much higher percentage as compared to the observations by Ashtekar et al.13In our present study, 7.2\% cases had history of prolonged rupture of membranes. This observation was comparable to that made by the study conducted by Rubatelli et al. (5.2\%).

In the present study majority of cases of respiratory distress were born of normal vaginal delivery followed by emergency caesarean section. Similar observations were also made by Levine et al ${ }^{14}$ and Liu et al. ${ }^{15}$

In the present study the case fatality rate of hyaline membrane disease was found to be the highest (50.8\%). Similar studies also had hyaline membrane disease with the highest case fatality rate. Case fatality rate of meconium aspiration syndrome was found to be $11.5 \%$ and that of pneumonia was found to be $20 \%$ which was similar to that observed by Rubetelli et al.

\section{CONCLUSION}

Meconium aspiration syndrome is the most common cause of respiratory distress among neonates born in Cheluvamba hospital followed by hyaline membrane disease. Neonatal respiratory distress had a male predilection. Maternal risk factors which were significant were primi parity, gestational diabetes, pre-eclampsia, multiple gestation, not receiving antenatal steroids in preterm labour. Intrapartum risk factors like prolonged labour, meconium stained amniotic fluid, mode of delivery, and low Apgar scores at birth also affect the aetiology of respiratory distress in neonates. Mortality among the neonates with respiratory distress was $24.8 \%$. The highest case fatality rate is for hyaline membrane disease $50.8 \%$.

\section{REFERENCES}

[1] Hermansen CL, Lorah KN. Respiratory Distress in the Newborn. Am Fam Physician 2007;76(7):987-94.

[2] Ersch J, Roth-Kleiner M, Baeckert P, et al. Increasing incidence of respiratory distress in neonates. Acta Pædiatrica 2007;96(11):1577-81.

[3] Report of the National Neonatal Perinatal Database (National Neonatology Forum) 2002-03.

[4] Abdelrahman SMK, Hamed SM, Nasr A. Neonatal respiratory distress in Omdurman Maternity Hospital, Sudan. Sudan J Paediatr 2014;14(1):65-70. 
[5] Santosh S, Kumar KK, Adarsha E. Clinical study of respiratory distress in newborn and its outcome. Indian Journal of Neonatal Medicine and Research 2013;1(1):2-4.

[6] Clark RH. The epidemiology of respiratory failure in neonates born at an estimated gestational age of 34 weeks or more. Journal of Perinatology 2005;25(4):251-7.

[7] Dutta A, Sinhamahapatra TK, Gayen S, et al. Spectrum of respiratory distress in newborn: a study from a tertiary care hospital in Kolkata. The Child and Newborn 2011;15(2):45-8.

[8] Kumar A, Bhat BV. Epidemiology of respiratory distress of newborns. Indian J Pediatr 1996;63(1):938.

[9] Mathur NB, Garg K, Kumar S. Respiratory distress in neonates with special reference to pneumonia. Indian Pediatrics 2002;39(6):529-37.

[10] Rubaltelli FF, Bonafe L, Tangucci $M$, et al. Epidemiology of neonatal acute respiratory disorders. A multicenter study on incidence and fatality rates of neonatal acute respiratory disorders according to gestational age, maternal age, pregnancy complications and type of delivery. Italian Group of Neonatal Pneumology. Biol Neonate 1998;74(1):7-15.
[11] Li-Ling Q, Cui-Qing L, Yun-Xia G, et al. Current status of neonatal acute respiratory disorders. Chin Med J 2010;123(20):2769-75.

[12] Dani C, Reali MF, Bertini G, et al. Risk factors for the development of respiratory distress syndrome and transient tachypnoea in newborn infants. Italian Group of Neonatal Pneumology. Eur Respir J 1999;14(1):155-9.

[13] Ashtekar SD, Ashtekar RS, Kumbhar SK, et al. Clinical study of meconium aspiration syndrome in relation to birth weight and gestational maturity at general hospital Sangli. Med Pulse - International Medical Journal 2014;1(5):189-92.

[14] Levine EM, Ghai V, Barton JJ, Strom CM. Mode of delivery and risk of respiratory diseases in newborns. Obstet Gynecol 2001;97(3):439-42.

[15] Liu J, Yang N, Liu Y. High-risk factors of respiratory distress syndrome in term neonates: a retrospective case-control study. Balkan Med J 2014;31(1):64-8. 